\title{
Structuration submicronique à grande échelle de surface de polymères à l'aide du rayonnement du laser à excimère
}

\author{
M. Bolle et S. Lazare
}

\section{Laboratoire de Photophysique et de Photochimie Moleculaire, URA 348 du CNRS, Université de Bordeaux I, 351 cours de la Libération, 33405 Talence, France}

Laser induced periodic structures of submicron dimensions can be obtained on polymer surfaces with polarized and low energy pulses of the excimer laser radiation. Full development of the structures requires 1000 pulses for a total dose of less than $10 \mathrm{~J} / \mathrm{cm}^{2}$ which can be delivered in a few seconds to surfaces which do not need to be carefuly aligned. Large scale treatments are therefore possible for thick polymer samples or for thin films used as resist on other materials surface.

L'absorption du faisceau polarisé et de faible fluence du laser à excimère par une surface de polymère produit une nouvelle classe de structures périodiques submicroniques ${ }^{1}$. Des rides espacées de moins de $0,2 \mu \mathrm{m}$ dans la direction du champ électrique sont obtenues avec la radiation ArF ou $\mathrm{KrF}$ lorsque celle-ci est fortement absorbée par le polymère. L'amplitude du relief périodique augmente avec le nombres d'impulsions utilisées, et un maximum est obtenu pour 1000 impulsions. De nombreux polymères en films minces ou épais, d'origine commerciale ou faits au laboratoire peuvent être structurés dans l'air ou dans le vide (ou dans les deux) suivant la structure chimique du polymere. La fluence nécessaire est située en dessous du seuil d'ablation ${ }^{2}$ (20 à 50 $\mathrm{mJ} / \mathrm{cm}^{2}$ ) et doit être choisie dans une fenêtre étroite $\left(3 \mathrm{a} 15 \mathrm{~mJ} / \mathrm{cm}^{2}\right)$ qui dépẹnd du polymère et de la longueur d'onde du laser. Le relief obtenu est observé par MEB, MET (Fig. 1) et caractérisé par ellipsométrie ${ }^{3}$. Les structures peuvent aussi être obtenues sur des films minces $(2000 \AA)$ déposés sur du silicium tel que sur la Fig.1a.

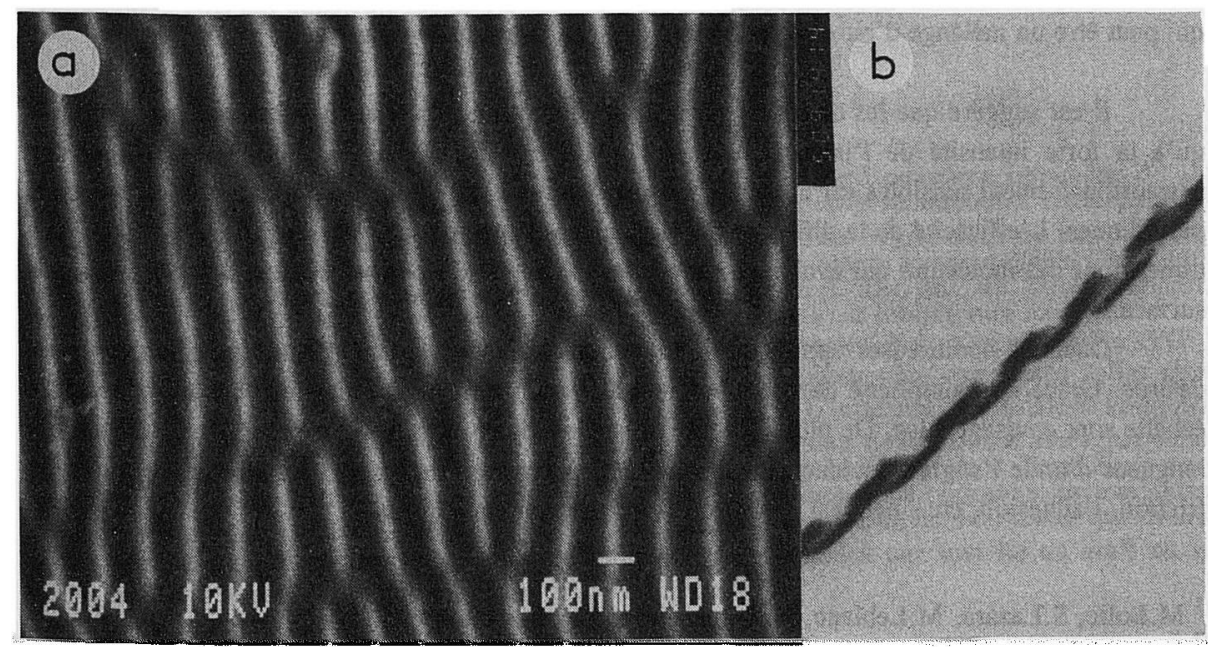

Fig.1 Structures périodiques réalisées dans un film de poly(éthylène téréphthalate) irradié à l'aide de 1000 impulsions $\left(3 \mathrm{~mJ} / \mathrm{cm}^{2}\right)$ à $193 \mathrm{~nm}$ du laser à excimère: a) vue au microscope électronique à balayage MEB, b) vue au microscope électronique à transmission MET; la couche métallique sombre a été déposée sur la surface après structuration pour une meilleure visualisation par MET. 
Le mécanisme de formation de ces structures, que nous étudions depuis récemment, fait appel à la formation d'onde de surface, qui résultent de la diffraction de l'impulsion incidente par les irrégularités de surface et se propagent parallelement à la surface. L'interférence de ces ondes avec $I_{0}$ provoque des maxima de champ espacés périodiquement sur la surface. Les ondes de surface sont décrites dans la littérature ${ }^{4}$ pour expliquer la formation de structures périodiques par irradiation de matériaux comme les métaux et les semi-conducteurs avec des lasers de plus grande longueur d'onde (Ruby, $\mathrm{Nd} / \mathrm{YAG}, \mathrm{CO}_{2}$ ). La nouveauté de ce travail vient des dimensions submicroniques dues à l'utilisation du rayonnement du laser à excimere de courte longueur d'onde et de la contribution de réactions photochimiques modifiant la surface des polymeres. Nous avons déterminé que, pour le polystyrène par exemple, la présence d'oxygène est indispensable au développement des structures. La contribution de l'onde de surface est mise en évidence par la dépendance angulaire de la période des structures indiquée a la Fig.2. Elle est décrite de façon univoque, par l'équation (I) donnant la période $\Lambda$ en fonction de l'angle d'incidence. Le modèle prévoit que l'onde diffusée traverse un milieu dont l'indice 1,39 est intermédiaire entre celui du vide et du polymère.
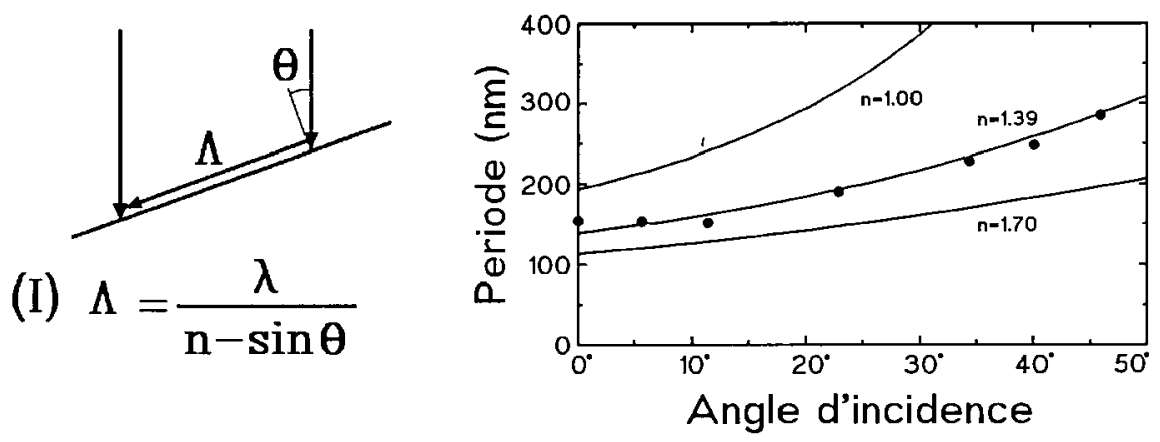

Fig.2 Période des structures en fonction de l'angle d'incidence, pour l'irradiation d'un film de PET $(n=1,700)$. La conclusion est que l'onde de surface se propage dans un milieu d'indice 1,39 qui peut être un mélange d'air et de polymère (couche de rugosité de surface).

Il est suggéré que les ondes de surface résultent d'un processus non-linéaire qui n'apparait qu'à la forte intensité de l'impulsion laser $\left(1 \mathrm{MW} / \mathrm{cm}^{2}\right)$. De plus les polymères se montrent extraordinairement sensibles car une dose modeste est nécessaire pour voir apparaître les structures périodiques. L'efficacité de la diffusion par des centres situés en surface pourrait être attribuée aux dimensions des molécules qui avoisinent celles des longueurs d'onde utilisées ou a des plasmons de surface.

Dans de nombreuses applications, une rugosité contrôlée d'amplitude submicronique est désirée. Grâce à la simplicité de notre processus d'irradiation les traitements de surface à grande échelle sont envisageables. De plus un contrôle sur les dimensions peut être exercé en changeant la longueur d'onde l'angle d'incidence et la nature du polymère. Des propriétés de surface comme la friction, l'adhésion, etc.. peuvent s'en trouver grandement améliorées.

${ }_{1}^{1}$ M.Bolle, S.Lazare, M.Leblanc, A.Wilmes Appl.Phys.Lett. 60, 674 (1992)

2 S. Lazare, V. Granier J.Appl.Phys. 63, 2110 (1988)

3 M.Bolle, S.Lazare soumis au J.Appl.Phys.

4 A.E.Siegman, P.M.Fauchet, IEEE J.Quant.Electron. QE-22, 1384 (1986); J.E.Sipe, J.F.Young, J.Preston and H.M. Driel, Phys.Rev. B 27, 1141 (1983) 